University of Nebraska - Lincoln

DigitalCommons@University of Nebraska - Lincoln

Faculty Publications, Department of Psychology

Psychology, Department of

March 1996

\title{
Envy and Jealousy as Discrete Emotions: A Taxometric Analysis
}

Nick Haslam

nhaslam@unimelb.edu.au

Brian H. Bornstein

University of Nebraska-Lincoln, bbornstein2@unl.edu

Follow this and additional works at: https://digitalcommons.unl.edu/psychfacpub

Part of the Psychiatry and Psychology Commons

Haslam, Nick and Bornstein, Brian H., "Envy and Jealousy as Discrete Emotions: A Taxometric Analysis" (1996). Faculty Publications, Department of Psychology. 189.

https://digitalcommons.unl.edu/psychfacpub/189

This Article is brought to you for free and open access by the Psychology, Department of at DigitalCommons@University of Nebraska - Lincoln. It has been accepted for inclusion in Faculty Publications, Department of Psychology by an authorized administrator of DigitalCommons@University of Nebraska - Lincoln. 


\title{
Envy and Jealousy as Discrete Emotions:
}

\section{A Taxometric Analysis ${ }^{1}$}

\author{
Nick Haslam ${ }^{2}$ \\ New School for Social Research
}

\author{
Brian H. Bornstein \\ Louisiana State University
}

Envy and jealousy may differ in kind or only by degree. In a study of emotion episodes recalled by 291 subjects, two forms of taxometric analysis were used to test between categorical and dimensional models of the two emotions. The two emotions yielded strong convergent evidence of discreteness, and commonly cooccurred. However, although subjects rated their episode to contain similar levels of "envy" and "jealousy," both terms were equally correlated with the presence of envy features and neither term was correlated substantially with the presence of jealousy features. Implications are drawn for the study of categorical distinctions between emotions, and for the relation between emotions and emotion terms.

Students of human emotion typically assume the existence of categorical distinctions between emotions corresponding to the terms that refer to them. According to this commonsense view of the relations of names to things, emotion terms have referents that differ in kind from one another. This "discontinuity" assumption undergirds theories of basic or discrete emotions (e.g., Ekman, 1992; Izard, 1993; Roseman, 1991). At the same time, however, there are accounts of the emotions that invoke continuous dimensions underlying affective experience (e.g., Russell, 1980; Watson \& Tellegen, 1985). These accounts imply that the emotional domain is not partitioned into discrete natural kinds. If there are no joints at which the domain can be

\footnotetext{
${ }^{1}$ The authors would like to thank Tammy Carter for assistance with data collection.

2 Address all correspondence concerning this paper to Nick Haslam, Department of Psychology, New School for Social Research, 65 Fifth Avenue, New York, New York 10003.
} 
carved, then any taxonomy must be somewhat artificial. Echoing these concerns, recent work in the cognitive structure of emotion warns against any simple conflation of emotion terms with the emotions themselves (Ortony, Clore, \& Collins, 1988); distinct terms may have indistinct referents. In response to these issues, the discreteness of emotions has begun to receive empirical attention (Etcoff \& Magee, 1992; Haslam, 1995).

The question of discreteness has recently been brought to the fore in discussions of envy and jealousy (Salovey, 1991). Parrott and Smith (1993) sought evidence for a "qualitative" distinction between the two emotions, following Salovey and Rodin's (1986) failure to find differences in the profile of their affective features. In two studies, Parrott and Smith found that when affective intensity was statistically controlled, and when envy- and jealousy-related situational elements were factorially manipulated, many differences could indeed be found. These differences corresponded closely to the traditional envy/jealousy distinction, according to which the former is related to social comparison and the latter to romantic rivalry (Sabini \& Silver, 1982; White, 1981): Feelings of inferiority, longing, and resentment were relatively more salient in envy, and feelings of distrust, anger, and fear of loss were more characteristic of jealousy.

Although Parrott and Smith's (1993) work demonstrated that the experiences of envy and jealousy are distinguishable, with distinctive features that compose orthogonal components, this does not in itself show that they represent discrete categories. For example, neuroticism and extraversion are distinct, orthogonal personality dimensions, but there is no evidence that there are discrete categories of neurotics and extraverts (Eysenck, 1967). Similarly, features of envy could covary independently of features of jealousy in emotion episodes marked by rivalry of some sort without there being bounded classes corresponding to two discrete emotions. In the same way that neurotic and extravert are used to refer to people who are relatively elevated on the continuous dimensions of neuroticism and extraversion, respectively, envy and jealousy might simply refer to emotion episodes that are elevated to a somewhat arbitrary extent on their respective dimensions. If this were true, then episodes of envy and jealousy, being drawn from the high end of different dimensions, would indeed show reliable differences in experiential quality, even if no category boundaries exist. For two entities to be truly discrete, that is, they must not only have distinctive features, but also show further evidence that these features form categories. Consequently, Parrott and Smith's findings of qualitative differences cannot fully answer the question of whether the terms envy and jealousy have discrete, categorical referents.

In order to show discreteness, then, an additional methodological and conceptual step must be taken. Having established that reliable differenc- 
es can be observed between two things, it remains to be determined whether these differences are latently matters of degree or matters of kind. To say that a difference is a matter of kind is to say that a latent discontinuity underlies manifest variation. If neuroticism were a matter of kind, for example, then two latent classes - one of neurotics and one of non-neurotics - would underlie manifest variation in it (i.e., scores on a neuroticism scale). If, on the other hand, neuroticism were a matter of degree, then there would be only one latent class, whose members vary continuously. As manifest variation may be continuous whether the latent situation is continuous or discontinuous, specialized methodologies are required to test between these alternatives. The present study attempted to perform such tests on the manifest differences between envy and jealousy demonstrated by Parrott and Smith (1993).

The existence of a latent discontinuity does not imply that meaningful variation is absent within each latent class or that there is no overlap between the two latent classes. Some men show more prototypically masculine traits than others, and some women show more such traits than some men, without there being any doubt that biological sex is discontinuous. Similarly, if jealousy is discrete then some episodes of jealousy will be more intense or more prototypically jealous than others, and some episodes of other emotions may well contain more features of jealousy than some episodes of jealousy. Nevertheless, discreteness would imply that jealousy is an affective kind to which emotion episodes categorically do or do not belong.

Discreteness also has implications for claims about the cooccurrence of emotions. Cooccurrence implies a conjunction of discrete entities or attributes which could occur separately or could both be absent. Neuroticism and extraversion, for instance, could not be said to cooccur in people unless there is a discrete class of people who are neurotic and an overlapping class of people who are extraverted. If neuroticism and extraversion are latently continuous attributes, then all people have degrees of both and cooccurrence would be meaningless. If envy and jealousy are not discrete, then emotion episodes could contain mixtures or blends of their respective features, but not the combined presence of two distinct affective syndromes. If envy and jealousy are not discrete, then, it is not clear in what sense they can be said to cooccur, as Parrott and Smith (1993) have claimed.

Arguments between discrete and dimensional accounts of latent variables are common in several domains of psychology (Meehl, 1992), and have important practical and ontological implications. However, statistical methods for adjudicating between them are only beginning to see widespread use, largely through the popularization of Meehl and Golden's (1982) taxometric methods. To date there have been several taxometric investigations of psychopathological syndromes ( e.g., Golden \& Meehl, 1979; Harris, Rice, 
\& Quinsey, 1994; Haslam \& Beck, 1994; Lenzenweger \& Korfine, 1992; Trull, Widiger, \& Guthrie, 1990; Tyrka, Haslam, \& Cannon, 1995) and personality variables (Gangestad \& Snyder, 1985; Strobe, 1989). Although all of these studies have tested for categories of people, taxometric analysis has also been used in a study of types of social relationship (Haslam, 1994). In a similar extension of taxometric analysis away from person types, it has recently been proposed as a valuable strategy for research on basic emotions and emotion families by Ekman and Davidson (1994). The present study represents the first foray of taxometric analysis into the study of emotion, and sought to illustrate the analysis for emotion researchers by answering the substantive question of the discreteness of envy and jealousy.

Taxometric methods test for the presence of latent categories or "taxa" in large samples, where each case is measured on one occasion. The most commonly employed taxometric procedure is the maximum covariance (or MAXCOV) method (Meehl \& Golden, 1982). The MAXCOV method uses multiple "indicator" variables that are hypothesized to discriminate a latent category. From this set of $N$ indicators all possible scales of $N-2$ indicators are then constructed, and the remaining two items are covaried among subsamples of cases along the scale (i.e., very low scorers, low scorers, intermediate scorers, and so on). Meehl and Golden showed that a function with maximum covariance in the intermediate range on the scale (strictly, at the point where there is maximum mixture of category members and nonmembers) and near-zero covariance at each endpoint (i.e. among subsamples with category members or nonmembers preponderating) implies a discrete latent variable. This pattern follows because variables that discriminate between categorical alternatives will tend to covary more between categories than within categories. For example, height and hair length are probabilistic indicators of biological sex among American adults, typically covarying in mixed groups. They do not strongly covary among women or among men, however, and will therefore covary maximally when an equal mixture of both is present. Such an equal mixture could be found by selecting cases from the intermediate range on a personality scale of masculinity-femininity, at the extremes of which cases would be disproportionately men and women.

Although the MAXCOV procedure has seen the most use in psychology, a variety of alternative taxometric and latent class procedures exist for testing discreteness. One of the more prominent is "commingling analysis" (MacLean, Morton, Elston, \& Yee, 1976), which has been used most in behavioral genetics (e.g., Price \& Stunkard, 1989). Rather than examining multiple indicator variables, like MAXCOV; commingling analysis examines single quantitative distributions, and tests whether observed distributions are best modeled by single- or multiple-component distributions. 
If multiple components are suspected - typically on the strength of skew and/or kurtosis in the observed distribution - commingling algorithms determine whether the fit of multiple-component models is significantly superior to the fit of single-component models, allowing statistically for the fact that adding components also adds degrees of freedom, and hence cannot result in inferior fit. If models containing multiple components show significantly superior fit, commingling analysis estimates their parameters. Commingling analysis of a mixed sample's distribution of scores on a masculinity-femininity scale, for example, would put a chi-square value to the superior fit of two-component versus one-component models, and estimate the mixing proportions of men and women in the sample and the difference between the means of the two component distributions in sample standard deviation units. In sum, commingling analysis represents a distinct, distribution-based form of analysis that complements the indicator-based MAXCOV procedure, and enables a convergent assessment of its findings.

The present study hoped to exploit the opportunities that the two forms of analysis provide for testing between discrete and continuous models of envy and jealousy. If the emotions prove to be discrete, the study sought to test how well subjects' usage of the two emotion terms corresponded to the two categories. The discreteness of the emotions themselves does not guarantee that people discriminate them in a corresponding manner, and it is possible that people's emotion concepts, and consequently their use of emotion terms, are systematically discrepant. Indeed, there is evidence that the usage of the two terms is somewhat ambiguous (Parrott \& Smith, 1993), and it is important to ask why this might be. The ambiguity could result from an imperfect mapping between the terms and the emotion categories, from cooccurrence of the emotion categories, from the emotions intergrading continuously, rather than being categories, from the emotion concepts being indistinct, or from some combination of these alternatives. Empirical analyses of discreteness, cooccurrence, and emotion labeling can help in assessing these alternatives.

In summary, the present study attempted to accomplish three goals: (1) to replicate Parrott and Smith's (1993) finding of distinctive features of envy and jealousy; (2) to examine taxometrically whether these features compose discrete categories; and (3) to examine how the terms envy and jealousy map onto the respective categories or dimensions. To these ends, the study generated a sample of first-person emotion episodes using an instruction that might equally elicit envy or jealousy but did not explicitly solicit either. Subjects rated these episodes on two sets of features that Parrott and Smith found to be distinctive to the two emotions, and also rated how "envious" and "jealous" they felt. Parrott and Smith's finding of qualitative differences between envy and jealousy would be replicated if the 
two feature sets each covaried systematically. If, in addition, both feature sets yielded taxometric categories (i.e., if episodes high in envy were categorically distinct from those in envy, and episodes high in jealousy were categorically distinct from those low in jealousy), then envy and jealousy would represent discrete emotions. That is, for envy and jealousy to be categorically different from one another, both must be categories with distinctive features, capable of being independently present or absent. ${ }^{3}$

\section{METHOD}

\section{Subjects}

Subjects were 291 undergraduates at Louisiana State University, who received extra credit for their participation. [Meehl and Golden (1982) reported that samples in the vicinity of 300 are generally ample for the use of taxometric procedures.] The sample included 221 women and 70 men, and the mean age was 19.8 years. Despite the large sample size, there were no sex differences on the 21 study items, except that women rated two items - "feeling threatened" and "guilt over feeling ill will"- higher at the .05 level. In view of the scarcity and small magnitude of these differences, and the absence of sex differences on the overall principal-component scores $[t(289)=0.125$ and $0.378, p s>.70]$, the results will be reported for the entire sample.

\section{Materials}

A two-part questionnaire form was constructed, in which subjects were required to describe a personal emotion episode in Part 1 and then to rate it on a series of 21 items in Part 2. Eighteen of the items were drawn from Parrott and Smith (1993).

Part 1 sought to elicit episodes that could equally fit standard descriptions of envy and jealousy, by explicitly mentioning neither term and using characteristics that they share. ${ }^{4}$ To this end, features determined by Parrott and Smith (1993) to be equally characteristic of envy and jeal-

\footnotetext{
${ }^{3}$ If envy and jealousy were mutually exclusive, and their episodes can be placed on the ends of a single bipolar scale, then their individual discreteness would imply their discreteness from one another. However, as the emotions are not exclusive, and have distinct unipolar factors underlying them (Parrott \& Smith, 19930, the discreteness of one from the other can only be inferred from the discreteness of each individually.

${ }^{4}$ If the terms envy and jealousy were used to solicit the emotion episodes, then spurious discontinuities could be produced by subjects selecting episodes from distinct regions within what is actually a latently continuous space. The assessment of the discreteness of the emotions would therefore be confounded by the discreteness of the emotion terms.
} 
ousy were combined with other common attributes drawn from Ortony et al. (1988) into the following instruction: "Please attempt to recall a time, as recently as possible, when you felt resentful, hostile or angry towards a 'rival' who seemed to be benefitting from some desirable event, and when you also felt hurt, insecure and unfairly treated by life." By incorporating the shared features of envy and jealousy, this instruction was sufficiently ambiguous to elicit either emotion, but not so ambiguous that it should elicit emotions markedly different from them. Other related emotions might be elicited by the instruction, and the study sought to determine whether envy and jealousy differed in kind from them. A brief narrative description of the episode was then solicited. Although these descriptions were made retrospectively, and hence may have been biased by expectations mediated by emotion concepts, we hoped to minimize such bias by sampling relatively recent emotion episodes and by excluding the terms envy and jealousy from the instructions.

Part 2 of the questionnaire contained a randomly ordered list of 18 items describing proposed discriminating features of envy and jealousy (nine items each), followed by three single items concerning self-rated envy, jealousy, and emotional intensity. Subjects were instructed to rate the emotional episode on all 21 items using 9-point Likert scales. All of the 18 envy and jealousy items were selected from Parrott and Smith's (1993) item sample using the following considerations: 91) Each item should empirically discriminate between envy and jealousy episodes in their first study, (2) each item should significantly load on the appropriate envy or jealousy component in their principal components analysis, and (3) items for each emotion should be drawn from different features clusters as much as possible, so as to minimize semantic redundancy. Consequently, the envy and jealousy item sets each contained items that were hypothesized to represent distinguishing, factorially pure, and nonredundant indicators of their respective emotions.

\section{Procedure}

Subjects completed the questionnaire forms in groups of 3 to 12 in a laboratory space, under the supervision of a research assistant. Completion typically took approximately $15 \mathrm{~min}$, after which subjects were debriefed.

\section{RESULTS}

The mean ratings of the 291 emotion episodes on the 9-point self-reported Envy and Jealousy scales (from 1, not at all characteristic, to 9, very characteristic) were $5.51(S D=3.06)$ and $5.64(S D=3.11)$, respec- 
tively, implying that many instances of both emotions were obtained. The mean rating on the 9-point intensity scale (from 1, not at all intense, to 9, extremely intense) was $6.94(S D=1.71)$, suggesting that these instances tended to be strong.

\section{Principal-Components Analysis}

Prior to performing the MAXCOV and commingling analyses, a principal-components analysis with varimax rotation was conducted on the 18 envy and jealousy items in order to replicate Parrott and Smith's (1993) finding of distinct, orthogonal envy and jealousy components and to test whether the items loaded as hypothesized on the respective components. Any item that failed to load uniquely on its component would be a poor indicator of its emotion and would therefore be excluded from the MAXCOV analysis, in the same way that any personality scale item that failed to load appropriately on the factor underlying the scale would be a poor indicator of the latent variable that the scale measures. In addition to replicating Parrott and Smith and winnowing the MAXCOV indicators, the principal-components analysis was performed to generate quantitative distributions of component scores for the commingling analyses, given that the components should discriminate their respective emotions. A two-component solution was recommended by scree test, and accounted for $40.4 \%$ of the variance in the item intercorrelation matrix. The component loadings of the 18 items are presented in Table I, along with the correlations of the three additional items with the respective component scores.

Table I strongly supports the existence of distinct envy and jealousy components, replicating Parrott and Smith's (1993) results to a striking degree. Eight of the nine hypothesized envy items loaded significantly (by convention $>.30$ ) on Component 1 and only one on Component 2 , and eight of the nine hypothesized jealousy items loaded significantly on Component 2 and only three on Component 1 . Feeling privately ashamed, like a failure and dissatisfied with oneself (E4, E8, and E9), appear to have been particularly distinctive (i.e., factorially pure) features of envy, while feeling betrayed, rejected, and distrustful (J1, J4, and J8) were particularly distinctive features of jealousy. One hypothesized envy item (E7-feeling degraded) was a better indicator of the jealousy component, and one hypothesized jealousy item (J6-feeling worried) is a better indicator of the envy component. Two further hypothesized jealousy items (J2-being afraid of a possible loss, and J5-feeling uncertain) loaded significantly on both components, and another (J3-feeling lonely) was factorially impure. 
Table I. Correlations of Envy (E) and Jealousy (J) Indicators and Self-Rated Emotion Features with the Two Principal-Components (Decimal Omitted)

\begin{tabular}{lcc}
\hline & Component 1 & Component 2 \\
\hline E1 Others would disapprove & $\mathbf{4 9}$ & 10 \\
E2 Longing for what other has & $\mathbf{6 0}$ & 07 \\
E3 Guilt over feeling ill will & $\mathbf{3 7}$ & 14 \\
E4 Feeling privately ashamed & $\mathbf{8 1}$ & -03 \\
E5 Motivated to improve self & $\mathbf{6 1}$ & -10 \\
E6 Feeling wishful & $\mathbf{3 3}$ & 12 \\
E7 Feeling degraded & 28 & $\mathbf{4 8}$ \\
E8 Feeling like a failure & $\mathbf{8 0}$ & 10 \\
E9 Feeling dissatisfied with self & $\mathbf{8 7}$ & -03 \\
J1 Feeling betrayed & -23 & $\mathbf{7 3}$ \\
J2 Being afraid of possible loss & $\mathbf{3 2}$ & $\mathbf{4 9}$ \\
J3 Feeling lonely & 27 & $\mathbf{5 7}$ \\
J4 Feeling rejected & 18 & $\mathbf{6 5}$ \\
J5 Feeling uncertain & $\mathbf{4 2}$ & $\mathbf{4 4}$ \\
J6 Feeling worried & $\mathbf{5 0}$ & 22 \\
J7 Feeling threatened & 15 & 58 \\
J8 Being distrustful & -14 & $\mathbf{6 8}$ \\
J9 Feeling vengeful & -05 & $\mathbf{5 9}$ \\
Self-rated envy & $54^{c}$ & 09 \\
Self-rated jealousy & $50^{c}$ & $\mathbf{1 2}$ \\
Self-rated intensity & $16^{b}$ & $49^{c}$ \\
\hline a $p$ < .05. & & \\
$b p<.01$. & & \\
$c p<.001$. & & \\
\end{tabular}

Consistent with the observation (Schoeck, 1969; Smith, Kim, \& Parrott, 1988) that in everyday usage jealousy has expanded into envy's traditional semantic territory, self-rated jealousy correlated as strongly with component 1 as self-rated envy. Interestingly, however, neither item correlated substantially with Component 2 (although self-rated jealousy narrowly reached significance), suggesting that, for this sample at least, episodes traditionally understood to involve jealousy were not discriminatively labeled as such. Apparently in moving into envy's semantic domain, jealousy to some degree abandoned its own. The two terms were used as virtual synonyms, in the sense of having a nearly identical reference, their items correlating .80 with one another $(p<.0001)$.

It is possible that the correlation of the two terms was artifactually high because the respective items were adjacent and in the same order (envy then jealousy) for all subjects, so that subjects might have anchored their jealousy ratings on their prior envy ratings. However, for this artifact to occur, subjects would have had to have very indistinct envy and jealousy concepts to enable the anchoring to take place at all. Moreover, such anchoring would have had to be so overwhelmingly strong as to make the correlation 
of jealousy with its component effectively zero, a result that could only occur if almost every subject anchored almost completely. It seems unlikely, then, that an artifactual explanation can account fully for the findings. However, it must be emphasized that the "synonymy" was obtained in an artificial task and for personal emotion episodes, and therefore can only be extended with caution to everyday emotion attribution and labeling.

In summary, the principal-components analysis indicated that distinct affective syndromes corresponding closely to traditional conceptions of envy and jealousy and to Parrott and Smith's (1993) factor structure persisted, and had further corollaries in emotional experience. For instance, the greater correlation of emotional intensity with Component 2 replicated earlier work indicating that jealousy is typically a stronger emotion than envy (Salovey \& Rodin, 1986; Smith et al., 1988). Nevertheless, the two affective syndromes were not distinctively labeled by their respective emotion terms.

\section{MAXCOV Analyses}

Prior to performing the MAXCOV analyses, it was necessary to select envy and jealousy items from the original set of 18 items that seemed to be good indicators of their respective emotions. Items that load weakly or not at all on their component probably measure another latent variable or contain substantial error, and items that load on both components contaminate the assessment of one with the other, so items with high and pure loadings are preferable. By analogy, if we wanted to test for a hypothesized category of introverts, we would want items that have been shown to correlate strongly with introversion, and which do not, like an item measuring social anxiety, also correlate with neuroticism.

Based on these considerations, six hypothesized envy items (E1, E2, E4, E5, E8, and E9) and five hypothesized jealousy items (J1, J4, J7, J8, and J9) were chosen as relatively pure and strong indicators of their components. The mean loadings of the envy items on the envy and jealousy components were +.70 and +.02 , respectively, whereas the mean loadings of the jealousy items were -.02 and +.65 . Therefore the item sets were highly distinctive to the two components, although the high loadings of the selected items, on the basis of which they were selected, would be expected to shrink somewhat on retesting (i.e., cross-validation).

Additive scales of all combinations of $N-2$ items (i.e., 15 four-item scales from the six envy items and 10 three-item scales from the five jealousy items) were constructed, and the two remaining items were covaried at decile ranges along their corresponding scales (e.g., E1 and E2 were covaried for the $10 \%$ of subjects scoring lowest on the scale of E4 + E5 + $\mathrm{E} 8+\mathrm{E} 9$, then for the next lowest $10 \%$, and so on). The covariances of the 


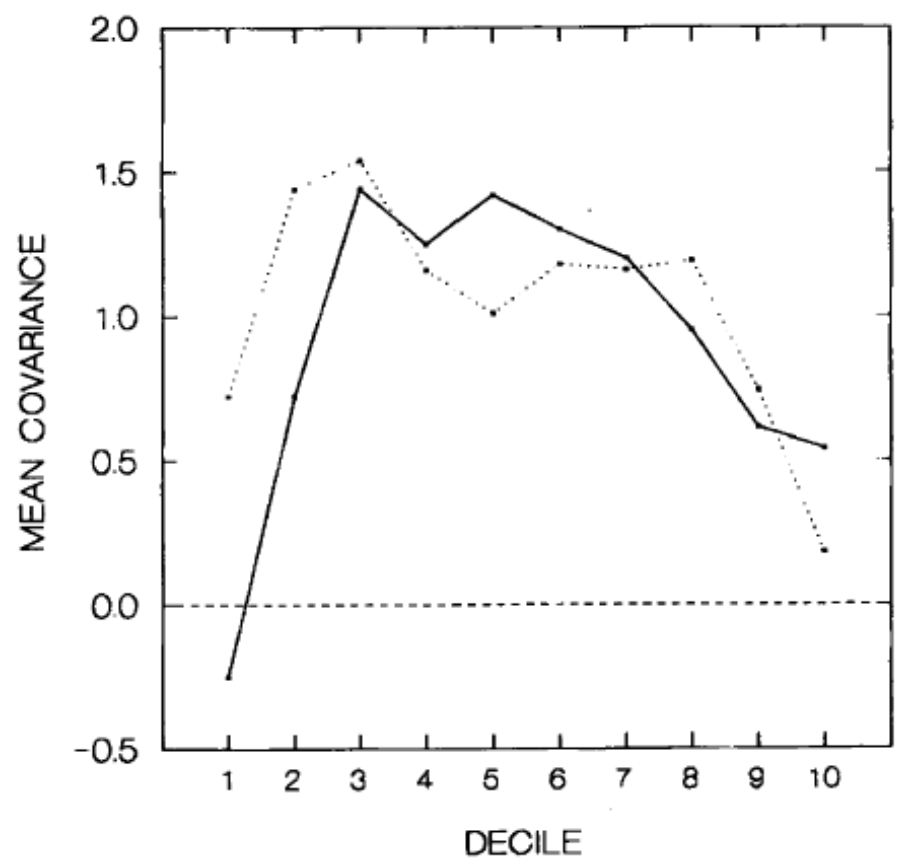

Fig. 1. Covariance plot for the envy (solid line) and jealousy (dotted line) indicators

envy and jealousy items, averaged over the 15 and 10 scales, respectively, and smoothed by running means are presented in Fig. 1.

Figure 1 displays clear elevations at intermediate ranges on the two covariance functions, consistent with the existence of discontinuity. Both emotions, that is, appear to be discrete kinds. The peak elevations on both functions are displaced somewhat to the left, indicating that the point of maximum mixture of category members and nonmembers was below the median of cases on the indicators. This finding implies in turn that the base rates of the two emotions (i.e., the proportions of the episode sample belonging to each emotion category) were greater than $50 \%$. Using a procedure described by Meehl and Golden (1982), these functions yielded base rate estimates of $71 \%$ for envy and $66 \%$ for jealousy. It is important to note that these estimates refer only to the proportions of emotion episodes within the sample, not to the proportions of such emotional events in the subjects' or the general population's lives outside of the study, or to proportions of types of people prone to the respective emotions.

The results of the MAXCOV analyses indicate that membership in the envy and jealousy categories was in an important sense not a matter of degree. This is not to say that some instances of jealousy, for instance, were not "better," more intense, or more prototypical than others. Better instanc- 
es would tend to have more or more elevated features of jealousy (i.e., fall in the higher deciles on the jealousy function) than worse instances, but discreteness implies that even these worse instances would be located inside the determinate boundaries of the jealousy category.

\section{Commingling Analyses}

Although the indicator-based MAXCOV analyses supported the discreteness of envy and jealousy, convergent evidence was sought from an alternative, distribution-based form of taxometric analysis. The SKUMIX program (MacLean et al., 1976) was used to implement a commingling analysis of the scores derived from the principal-components analysis. These scores represented the position of the emotion episodes on the two components, based on weighted sums of all 18 envy and jealousy items. If the two emotions were discrete, then the observed distributions of scores on the two components should each contain two latent distributions, a relatively high scoring distribution of emotion members and a relatively low scoring distribution of nonmembers. If, on the other hand, the emotions were dimensional, a single latent distribution should model the observed distributions satisfactorily. The commingling analyses therefore tested whether these observed distributions were modeled better by one or two latent distributions. ${ }^{5}$

Superficial inspection of the distributions of component scores suggested the possibility of more than one latent distribution. Both distributions were slightly negatively skewed and significantly $(p<.01)$ platykurtic (short-tailed), as would be expected if two overlapping distributions were combined, with the high-scoring distribution being larger. In order to assess this possibility, four models of each component score distribution were tested, in each of which some parameters were fixed and others free to vary. Parameters were the overall mean of the distribution $(u)$; the variance of the distributions $(V)$, constrained to be equal for two-distribution models; skewness $(p)$, similarly constrained to equality for two-distribution models; displacement $(t)$, the separation in standard deviation units of latent distribution means; and mixing proportion $(q)$, the base rate of the upper distribution. The four models were as follows:

1. One normal distribution [ $u$ and $V$ free, $p$ fixed to 1 (no skew), $t$ and $q$ fixed to zero]

2. One skewed distribution ( $u, V$, and $p$ free, $t$ and $q$ fixed to zero)

3. Two normal distributions ( $u, V, t$, and $q$ free, $p$ fixed to 1$)$

4. Two skewed distributions ( $u, V, p, t$, and $q$ free)

${ }^{5}$ Commingling analysis can test three distribution models, but these were not relevant to the research question of discreteness versus continuity. 
Table II. Parameter Estimates and Goodness-of-Fit Values for the Commingling Analyses of the Envy Component $(u=$ Grand Mean, $V=$ Variance of distributions, $p=$ Skewness, $t=$ Separation of distribution means, $q=$ Upper Distribution Base Rate, $-2 \ln L=$ Fit Statistic; Fixed Parameters in Parentheses)

\begin{tabular}{lccccccc}
\hline & \multicolumn{7}{c}{ Parameters } \\
\cline { 2 - 8 } Model & $u$ & $V$ & $p$ & $t$ & $q$ & Free & -2 In $L$ \\
\hline 1 (one normal) & 0.00 & 1.00 & $(1.00)$ & $(0.00)$ & $(0.00)$ & 2 & 824.85 \\
2 (one skewed) & 0.02 & 0.99 & 1.22 & $(0.00)$ & $(0.00)$ & 3 & 824.46 \\
3 (two normal) & 0.00 & 0.36 & $(1.00)$ & 1.61 & 0.75 & 4 & 799.06 \\
4 (two skewed) & -0.01 & 0.36 & 0.88 & 1.62 & 0.76 & 5 & 798.97 \\
\hline
\end{tabular}

The SKUMIX program provided a measure of goodness of fit for each model expressed as $-2 \ln L$ (minus twice the natural logarithm of the likelihood). The difference in this quantity between two models is distributed as chi square with degrees of freedom equal to the difference in number of free parameters. Results for the four models are presented in Tables II and III.

Tables II and III present very similar patterns of findings. In both cases, relaxing the skewness parameter $p$ did not significantly improve the fit of either the one- or two-distribution models [Models 2 vs. 1 and 4 vs. 3, respectively; $\chi^{2}(1)=0.09-1.52$, n.s.]. Consequently the unskewed models $(1$ and 3 ) provided satisfactory estimates of the fit of one- and two-distribution solutions. Comparison of these two models revealed the clear superiority of the two-distribution solution for envy $\left[\chi^{2}(2)=25.79, p<.0001\right]$ and for jealousy $\left[\chi^{2}(2)=9.14, p<.01\right]$. The commingling analyses therefore replicated the MAXCOV findings of discreteness. According to the parameter estimates derived from Model 3, discrete envy and jealousy categories existed with base rates (parameter $q$ ) of $75 \%$ and $76 \%$ in the sample of emotion episodes. These estimates were reasonably close to those derived from the MAXCOV analyses ( $71 \%$ and $66 \%$, respectively). Given that the principal-component scores were uncorrelated, these estimates imply that

Table III. Parameter Estimates and Goodness-of-Fit Values for the Commingling Analyses of the Jealousy Component $(u=$ Grand Mean, $V=$ Variance of Distributions, $p=$ Skewness, $t=$ Separation of Distribution Means, $q=$ Upper Distribution Base Rate, $-2 \ln L=$ Fit Statistic; Fixed Parameters in Parentheses)

\begin{tabular}{lccccccc}
\hline & \multicolumn{8}{c}{ Parameters } \\
\cline { 2 - 8 } Model & $u$ & $V$ & $p$ & $t$ & $q$ & Free & $-2 \ln L$ \\
\hline 1 (one normal) & 0.00 & 1.00 & $(1.00)$ & $(0.00)$ & $(0.00)$ & 2 & 824.84 \\
2 (one skewed) & 0.03 & 0.98 & 1.33 & $(0.00)$ & $(0.00)$ & 3 & 823.79 \\
3 (two normal) & 0.00 & 0.49 & $(1.00)$ & 1.45 & 0.76 & 4 & 815.70 \\
4 (two skewed) & 0.09 & 0.44 & 2.05 & 1.51 & 0.62 & 5 & 814.18 \\
\hline
\end{tabular}


$57 \%(.75 * .76)$ of the sample of emotion episodes represented cooccurrences of envy and jealousy, consistent with Parrott and Smith's (1993) claim that cooccurrence is common. Similarly, the base rate estimates suggest that only $6 \%(.25 * .24)$ of the sample exemplified neither emotion, implying that the instruction was successful in eliciting the two emotions. As the principal-components analysis was constrained to produce orthogonal components, these cooccurrence and nonoccurrence estimates may be somewhat inaccurate. However, supplementary analyses using oblique rotation indicated that the correlation of the two components was effectively zero, suggesting that the $6 \%$ figure was reasonably accurate.

\section{DISCUSSION}

The findings of the present study indicate that envy and jealousy can be differentiated. The evidence of the principal-components analysis strikingly replicated Parrott and Smith's (1993) findings concerning distinctive features. In addition, two convergent analyses indicated that the distinctive features of the two emotions compose discrete, bounded categories that correspond closely to traditional definitions. envy is characterized by feelings of shame, failure, dissatisfaction with self, and longing for what another had, whereas jealousy is characterized by feelings of betrayal, distrust, rejection, threat and loneliness. Envy and jealousy appear to be complex affective kinds that are distinct but often combined elements of the emotional repertoire. They do not appear to be conventionally designated regions in a continuous affective domain.

Although the present study demonstrated discrete categories of emotional experience corresponding to traditional descriptions of envy and jealousy, it also indicated that these categories do not directly correspond to the everyday usage of the respective terms. Envy and jealousy were used almost synonymously - although a methodological artifact may have contributed to this - and referred preferentially to the envy category. These results replicated the robust finding of a historical expansion in the reference of jealousy to encompass envy (e.g., Schoeck, 1969), but suggest that in this sample at least a further shift may have occurred. Rather than envy covering only a subset of jealousy's referential range, both terms now appear to have taken root in the envy category.

It remains to be explained why jealousy, having expanded its range to refer to a new category, should withdraw from the category in which it was originally anchored. It may be that the first shift has proceeded to the point where jealousy's dominant usage is now in reference to traditional envy, and that distinct secondary usages, such as jealousy referring to traditional jealousy, have tended to erode. Although this explanation is highly spec- 
ulative, a similar phenomenon is exemplified by a historical shift in the meaning of anger. Originally referring to feelings of distress or affliction, and derived from an Old Norse word meaning grief, it acquired the sense of rage in the 13th century (Ayto, 1991). This sense has subsequently become dominant, and the previous senses have vanished. By this kind of account, there may now be a tendency for episodes of jealousy to be designated by other emotion terms, rather than by jealousy, because the latter now prototypically refers to something that differs in its feel, its situational determinants, and its moral implications (Sabini \& Silver, 1982; Schoeck, 1969). This claim could be tested by a study of the unconstrained labeling of naturally occurring emotion episodes, with the prediction that independently rated envy episodes would be labeled jealousy more often than envy, and independently rated jealousy episodes would most commonly receive labels other than jealousy.

Whatever the explanation of the variant senses of envy and jealousy, the present study cautions strongly against assuming any straightforward relationship between emotion terms and emotions themselves (cf. Ortony et al., 1988). The usage of emotion terms may be susceptible to historical changes that largely spare their original affective referents. It is quite conceivable that historical changes are expressed most directly in altered labeling practices, and that the designated experiences either lag behind in their alterations or remain substantially unchanged. Although the position that unanchored emotion labels drift over relatively changeless emotional kinds is consistent with theories of universal or basic emotions ( e.g., Ekman, 1992) and antithetical to most social constructionist theories (e.g., Harré, 1986), it can also be made consistent with a sophisticated, culturally informed theory. Greenwood (1994) has recently argued that emotions are constituted by social forms of evaluation of actions and social relations, rather than by discourse about emotions that explicitly entertains emotion labels. Such labels are not necessary for the sort of moral commentary out of which cultural meaning is given to emotional life, and out of which it may be transformed. By this "realist" account, emotion labels play no direct causal role in the transformation of emotions. Although the present study cannot adjudicate between universalist and realist theories, it is important to note that its findings concerning the apparently greater stability of emotions than their labels do not entail a nonsocial, universalist interpretation.

The present study's finding of discreteness supports earlier work claiming the existence of category boundaries in the field of emotion. Category boundaries had previously been demonstrated in the perception of emotion (Etcoff \& Magee, 1992) and in its mental representation (Haslam, 1995), and it appears that the experience of emotion can now 
be added. It might also be that the situational determinants of emotions are discrete, a position that Roseman's (1991) work on the appraisal dimensions of discrete emotions embodied. This position has been argued by Salovey and Rodin (1986) in relation to envy and jealousy, although the present study indicates that situational differences do not exhaust the distinctiveness of the two emotions.

Indeed the discreteness of the two emotions might flow from the distinctness and specificity of the situations of adaptive functions that call them into play. Discrete affective responses (R) are most likely to stem from discrete eliciting conditions $(\mathrm{C})$, without the categorical presence of which the response is not triggered (i.e., if $\mathrm{C}$ then $\mathrm{R}$; if not- $\mathrm{C}$ then not$\mathrm{R})$. On the other hand, continuously variable responses are more likely to stem from more diffuse and graduated conditions (i.e., the more $\mathrm{C}$ or $\mathrm{Cs}$ are present, the more $\mathrm{R}$ is elicited). By this account, the discreteness of envy and jealousy suggests that they have discrete eliciting conditions or, alternatively, discrete adaptive functions that make particular situations capable of triggering them. Precisely what such conditions or functions might be remains to be determined.

This study's convergent evidence for category boundaries in emotion is also consistent with Wierzbicka's (1992) claim that emotion concepts may be definable, although it does not entail it. As the present study suggests, it is crucial in the affective domain to distinguish between emotion concepts and the emotions themselves. It is conceivable that emotion concepts might be sharp and definable and yet impose arbitrary divisions on emotional phenomena that lack real discontinuities, just as it is conceivable that people mentally represent emotions such as jealousy as graded prototype structures (Rosch \& Mervis, 1975; Sharpsteen, 1993) although the phenomena themselves are in some sense discrete. In this light, the common practice of invoking "fuzzy" boundaries begs the question of discreteness because there are two senses of "fuzziness." In one sense, fuzziness is an intrinsic property of categories, and in the other it is a matter of the concept-mediated identifiability of category members.

In the second sense of fuzziness, a category is fuzzy because its members and nonmembers overlap phenotypically and our concepts sometimes assign degrees of membership or prototypically to potential members. Such overlap may make it impossible to determine to which class potential members belong, although discrete classes unquestionably exist. For example, our concepts of obesity may consist of probabilistic feature lists that enable graded typicality judgments about individual cases, although there appears to be a discrete class of obese people whose weight overlaps the distribution of its nonmembers (Price \& Stunkard, 1989). In the first sense of the term, however, fuzziness is intrinsic to categories. For example, the cate- 
gory of tall people does not correspond to a genotypically distinct population, and people may therefore belong to it by degrees according to the arbitrary conventions that our concepts impose on the height continuum. The present study argues that envy and jealousy are not fuzzy categories in this sense; rather, instances of both belong to discrete but phenotypically overlapping classes, making identification of marginal cases very difficult. People's concepts of envy and jealousy may be intrinsically fuzzy, assigning emotion episodes to the respective prototypes by degrees, but the categories that ground these concepts are not so.

Although much remains to be done in accounting theoretically for the bases of discrete categories, demonstrating their existence is itself a significant step. First, by locating nature's joints (Gangestad \& Snyder 1985) it works against the conflation of emotions that are in some ways alike, enabling scientific advances by unconfounding discrete phenomena. Second, it stimulates study of the undergirding of the category boundaries, such as necessary features or discrete cultural schemata, eliciting conditions or physiological mechanisms. Third, it places the phenomenon under investigation on a more solid ontological footing. A latent discontinuity implies a singular, nonarbitrary class of instances having something in common and a determinate boundary, whereas a latent continuity implies instances that can only be linguistically marked by more or less arbitrary convention. In view of these advantages, the further investigation of categorical distinctions in the field of emotion is clearly warranted.

\section{REFERENCES}

Ayto, J. (1991). Dictionary of word origins. New York: Arcade.

Ekman, P. (1992). An argument for basic emotions. Cognition and Emotion, 6, 169-200.

Ekman, P., \& Davidson, R. J. (1994). Affective science: A research agenda. In P. Ekman \& R. J. Davidson (Eds.), The nature of emotion: Fundamental questions (pp. 411-430). New York: Oxford University Press.

Etcoff, N. L., \& Magee, J. J. (1992). Categorical perception of facial expressions. Cognition, 44, $227-240$.

Eysenck, H. J. (1967). The biological basis of personality. Springfield, IL: Thomas.

Gangestad, S. W., \& Snyder, M. (1985). "To carve nature at its joints": On the existence of discrete classes in personality. Psychological Review, 92, 317-349.

Golden, R. R., \& Meehl, P. E. (1979). Detection of the schizoid taxon with MMPI indicators. Journal of Abnormal Psychology, 88, 217-233.

Greenwood, J. D. (1994). Realism, identity and emotion. London: Sage.

Harré, R. (1986). An outline of the social constructionist movement. In R. Harré (Ed), The social construction of emotions (pp. 1-14). Oxford, England: Blackwell.

Harris, G. T., Rice, M. E., \& Quinsey, V. L. (1994). Psychopathy as a taxon: Evidence that psychopaths are a discrete class. Journal of Consulting and Clinical Psychology, 62, 387-397.

Haslam, N. (1994). Categories of social relationship. Cognition, 53, 59-90. 
Haslam, N. (1995). The discreteness of emotion concepts: Categorical structure in the affective circumplex. Personality and Social Psychology Bulletin, 21, 1012-1019.

Haslam, N., \& Beck, A. T. (1994). Subtyping major depression: A taxometric analysis. Journal of Abnormal Psychology, 103, 686-692.

Izard, C. E. (1993). Organizational and motivational functions of discrete emotions. In M. Lewis \& J. M. Haviland (Eds.), Handbook of emotions (pp. 631-641). New York: Guilford Press.

Lenzenweger, M. F., \& Korfine, L. (1992). Confirming the latent structure of schizotypy: A taxometric analysis. Journal of Abnormal Psychology, 101, 567-571.

MacLean, C. J., Morton, N. E., Elston, R. C., \& Yee, S. (1976). Skewness in commingled distributions. Biometrics, 32, 695-699.

Meehl, P. E. (1992). Factors and taxa, traits and types, differences of degree and differences in kind. Journal of Personality, 60, 117-174. .

Meehl, P. E., \& Golden, R. R. (1982). Taxometric methods. In E C. Kendall \& J. N. Butcher (Eds.), Handbook of research methods in clinical psychology (pp. 127-181). New York: Wiley.

Ortony, A., Clore, G. L., \& Collins, A. (1988). The cognitive structure of emotions. Cambridge, England: Cambridge University Press.

Parrott, W. G., \& Smith, R. H. (1993). Distinguishing the experiences of envy and jealousy. Journal of Personality and Social Psychology, 64, 906-920.

Price, R. A., \& Stunkard, A. J. (1989). Commingling analysis of obesity in twins. Human Heredity, 39, 121-135.

Rosch, E., \& Mervis, C. (1975). Family resemblances: Studies in the internal structure of categories. Cognitive Psychology, 7, 573-605.

Roseman, I. J. (1991). Appraisal determinants of discrete emotions. Cognition and Emotion 5, 161-200.

Russell, J. A. (1980). A circumplex model of affect. Journal of Personality and Social Psychology, 39, 1161-1178.

Sabini, J., \& Silver, M. (1982). Moralities of everyday life. New York: Oxford University Press.

Salovey, P., \& Rodin, J. (1986). The differentiation of social-comparison jealousy and romantic jealousy. Journal of Personality and Social Psychology, 50, 1100-1112.

Schoeck, H. (1969). Envy: A theory of social behavior. New York: Harcourt, Brace \& World

Sharpsteen, D. J. (1993). Romantic jealousy as an emotion concept: A prototype analysis. Journal of Social and Personal Relationships, 10, 69-82.

Smith, R. H., Kim, S. H., \& Parrott, W. G. (1988). Envy and jealousy: Semantic problems and experiential distinctions. Personality and Social Psychology Bulletin, 14, 401-409.

Strube, M. J. (1989). Evidence for the type in type A behavior: A taxometric analysis. Journal of Personality and Social Psychology, 56, 972-987.

Trull, T. J., Widiger, T. A., \& Guthrie, P. (1990). Categorical versus dimensional status of borderline personality disorder. Journal of Abnormal Psychology, 99, 40-48.

Tyrka, A., Haslam, N., \& Cannon, T. D. (1995). Detection of a longitudinally-stable taxon of individuals at risk for schizophrenia spectrum disorders. In A. Raine, T. Lencz, \& S. A. Mednick (Eds.), Schizotypal personality disorder. New York: Cambridge University Press.

Watson, D., \& Tellegen, A. (1985). Toward a consensual structure of mood. Psychological Bulletin, 98, 219-235.

White, G. L. (1981). A model of romantic jealousy. Motivation and Emotion, 5, 295-310.

Wierzbicka, A. (1992). Defining emotion concepts. Cognitive Science, 16, 539-581. 